PROCEEDINGS OF THE

AMERICAN MATHEMATICAL SOCIETY

Volume 140, Number 6, June 2012, Pages 1897-1907

S 0002-9939(2011)11059-1

Article electronically published on October 3, 2011

\title{
ON GORENSTEIN INJECTIVITY OF TOP LOCAL COHOMOLOGY MODULES
}

\author{
TAKESHI YOSHIZAWA
}

(Communicated by Bernd Ulrich)

\begin{abstract}
R. Sazeedeh showed that top local cohomology modules are Gorenstein injective in a Gorenstein local ring with at most two dimensions. In this paper, it is proved that the condition of dimension in his result cannot be relaxed and the conclusion in his result holds for complete local hypersurface rings with any dimension.
\end{abstract}

\section{INTRODUCTION}

Through this paper, all rings are commutative Noetherian and all modules are unitary. All maximal Cohen-Macaulay modules considered here will be finitely generated.

The notion of Gorenstein injective modules was introduced by E. E. Enochs and O. M. G. Jenda in [6]. The class of Gorenstein injective modules is greater than the class of injective modules. Gorenstein injective modules, or more extensively Gorenstein injective dimension, are actually studied by many authors. See, for example, [8, 11, 13]. Recently, in [13, R. Sazeedeh showed the following result: if $R$ is a Gorenstein local ring with at most two dimension, then the top local cohomology module $H_{J}^{\operatorname{dim} R}(R)$ is a Gorenstein injective $R$-module for any ideal $J$ of $R$. Therefore it is natural to ask whether the conclusion in his result holds for a Gorenstein local ring with arbitrary dimension. But it seems difficult to decide whether modules are Gorenstein injective from the definition immediately. Thus, to study the above problem, we shall try to find a practical way of concluding that top local cohomology modules are Gorenstein injective.

Our strategy is the following. P. Schenzel proved the existence of a monomorphism from the Matlis dual module of the top local cohomology module to the canonical module in [16]. R. Takahashi, Y. Yoshino and the author introduced a notion of generalized local cohomology modules associated to a pair of ideals and showed that the image of the above monomorphism is isomorphic to the generalized local cohomology module in [19. By using these results, the problem comes down to a question of whether generalized local cohomology modules are maximal Cohen-Macaulay modules. We can give an example of a non-Gorenstein injective top local cohomology module over a 3-dimensional Gorenstein local ring and show

Received by the editors January 4, 2010 and, in revised form, October 11, 2010 and February 5, 2011.

2010 Mathematics Subject Classification. Primary 13D05, 13D45.

Key words and phrases. Local cohomology, Gorenstein injective module.

(C)2011 American Mathematical Society Reverts to public domain 28 years from publication 
that the conclusion in R. Sazeedeh's result is valid for complete local hypersurface rings with any dimension.

The organization of this paper is as follows.

We recall some basic concepts and properties of Gorenstein injective and strongly cotorsion modules in section 1.

In section 2, we study the relationship between Gorenstein injective modules, strongly cotorsion modules and local cohomology modules.

In section 3, we shall show that any top local cohomology module over a complete local hypersurface ring is Gorenstein injective (Theorem 3.5) and give an example of a non-Gorenstein injective top local cohomology module over a 3-dimensional Gorenstein local ring (Example 3.7).

\section{Preliminaries}

Let us recall some definitions of modules. J. Xu introduced notions of strongly cotorsion modules and strongly torsion free modules in [20].

Definition 1.1. (1) An $R$-module $M$ is called strongly cotorsion if $\operatorname{Ext}_{R}^{1}(X, M)=0$ for all $R$-modules $X$ of finite flat dimension.

(2) An $R$-module $M$ is called strongly torsion free if $\operatorname{Tor}_{1}^{R}(X, M)=0$ for all $R$-modules $X$ of finite flat dimension.

E. E. Enochs and O. M. G. Jenda introduced notions of Gorenstein injective modules and Gorenstein projective modules in [6] and of Gorenstein flat modules together with B. Torrecillas in 7].

Definition 1.2. (1) An $R$-module $M$ is called Gorenstein injective if there is an exact sequence

$$
\cdots \rightarrow E_{1} \rightarrow E_{0} \rightarrow E^{0} \rightarrow E^{1} \rightarrow \cdots
$$

of injective modules such that $M=\operatorname{Ker}\left(E^{0} \rightarrow E^{1}\right)$ and such that $\operatorname{Hom}_{R}(E,-)$ leaves the above sequence exact whenever $E$ is an injective $R$-module.

(2) An $R$-module $M$ is called Gorenstein projective if there is an exact sequence

$$
\cdots \rightarrow P_{1} \rightarrow P_{0} \rightarrow P^{0} \rightarrow P^{1} \rightarrow \cdots
$$

of projective modules such that $M=\operatorname{Ker}\left(P^{0} \rightarrow P^{1}\right)$ and such that $\operatorname{Hom}_{R}(-, P)$ leaves the above sequence exact whenever $P$ is a projective $R$-module.

(3) An $R$-module $M$ is called Gorenstein flat if there is an exact sequence

$$
\cdots F_{1} \rightarrow F_{0} \rightarrow F^{0} \rightarrow F^{1} \cdots
$$

of flat modules such that $M=\operatorname{Ker}\left(F^{0} \rightarrow F^{1}\right)$ and such that $E \otimes_{R}$ - leaves the above sequence exact whenever $E$ is an injective $R$-module.

Remark 1.3. (1) Any injective module is a strongly cotorsion module and a Gorenstein injective module.

(2) If $R$ is $n$-Gorenstein, that is, $R$ is Gorenstein with $\operatorname{dim} R=n$, then strongly cotorsion modules are precisely Gorenstein injective modules.

(3) In a Cohen-Macaulay local ring, maximal Cohen-Macaulay modules are precisely strongly torsion free finitely generated modules. (See $3,4,9$.)

The following theorem was proved by R. Sazeedeh. We shall refer to this theorem as R. Sazeedeh's Theorem. 
Theorem 1.4 ([13, Theorem 2.6], [15, Theorem 2.15]). Let $(R, \mathfrak{m})$ be a CohenMacaulay local ring of dimension $d, J$ be a nonzero ideal of $R$ and $M$ be a maximal Cohen-Macaulay $R$-module. If $d \leq 2$, then $H_{J}^{d}(M)$ is strongly cotorsion. In particular, if $R$ is Gorenstein and $d \leq 2$, then $H_{J}^{d}(R)$ is Gorenstein injective.

\section{Characterization of Gorenstein Ring by properties OF LOCAL COHOMOLOGY MODULES}

In this section, we shall give a characterization of a Gorenstein ring by Gorenstein injectivity of local cohomology modules and make a close examination of R. Sazeedeh's Theorem.

Throughout the rest of this paper, let $(R, \mathfrak{m})$ be a local ring with a maximal ideal $\mathfrak{m}$ and $E_{R}(R / \mathfrak{m})$ be the injective hull of $R / \mathfrak{m}$. The functor $\operatorname{Hom}_{R}\left(-, E_{R}(R / \mathfrak{m})\right)$ is denoted by $(-)^{\vee}$. For a ring $R$ admitting the dualizing complex $D_{R}$, we denote by $K_{M}$ the canonical module of an $R$-module $M$, which is defined to be

$$
K_{M}=H^{d-t}\left(\mathbf{R H o m}_{R}\left(M, D_{R}\right)\right),
$$

where $d=\operatorname{dim} R$ and $t=\operatorname{dim} M$.

Let us recall the definition of local cohomology functors associated to a pair of ideals which was defined in [19] as the generalization of local cohomology functors with closed support. (As concerns the ordinary local cohomology functor, the reader should consult [2].)

Definition 2.1. Let $I$ and $J$ be ideals of $R$. We set

$$
W(I, J)=\left\{\mathfrak{p} \in \operatorname{Spec}(R) \mid I^{n} \subseteq \mathfrak{p}+J \text { for some integer } n>0\right\} .
$$

For an $R$-module $M, \Gamma_{I, J}(M)$ denotes a submodule of $M$ consisting of all elements of $M$ with support in $W(I, J)$, that is,

$$
\Gamma_{I, J}(M)=\{x \in M \mid \operatorname{Supp}(R x) \subseteq W(I, J)\} .
$$

The left exact functor $\Gamma_{I, J}$ is called an $(I, J)$-torsion functor. We denote by $H_{I, J}^{i}$ the $i$ th right derived functor of $\Gamma_{I, J}$ and refer to it as the $i$ th local cohomology functor with respect to $(I, J)$.

Remark 2.2. Let $(R, \mathfrak{m})$ be a local ring and $J$ be an ideal of $R$. For a prime ideal $\mathfrak{p}$ of $R, \mathfrak{p} \in W(\mathfrak{m}, J)$ if and only if $\mathfrak{p}+J$ is an $\mathfrak{m}$-primary ideal.

First of all, we shall show the following proposition, which is the base of this paper.

Proposition 2.3. Let $(R, \mathfrak{m})$ be a complete Cohen-Macaulay local ring, $J$ be an ideal of $R$ and $M$ be a finitely generated $R$-module of dimension $t$. Then the following conditions are equivalent:

(1) $H_{J}^{t}(M)$ is a strongly cotorsion $R$-module;

(2) $\Gamma_{\mathfrak{m}, J}\left(K_{M}\right)$ is a maximal Cohen-Macaulay $R$-module or zero.

Proof. Since $H_{J}^{t}(M)$ is an Artinian $R$-module by [2, 7.1.6 Theorem], we have $H_{J}^{t}(M) \cong H_{J}^{t}(M)^{\vee \vee}$. By [14, Lemma 2.1], it follows that $H_{J}^{t}(M)$ is strongly cotorsion if and only if $H_{J}^{t}(M)^{\vee}$ is strongly torsion free. Here, we note that $H_{J}^{t}(M)^{\vee} \cong \Gamma_{\mathfrak{m}, J}\left(K_{M}\right)$ by [19, Theorem 5.11] and this is a finitely generated $R$ module. Therefore, it follows that $H_{J}^{t}(M)^{\vee}$ is strongly torsion free if and only if $\Gamma_{\mathfrak{m}, J}\left(K_{M}\right)$ is a maximal Cohen-Macaulay $R$-module. (See Remark 1.3 (3).) 
In particular, we can see the following result, which has already been seen in 15.

Corollary 2.4. Let $(R, \mathfrak{m})$ be a complete Cohen-Macaulay local ring of dimension $d$. Then $H_{\mathfrak{m}}^{d}(R)$ is a strongly cotorsion $R$-module.

Remark 2.5. Let $(R, \mathfrak{m})$ be a Cohen-Macaulay local ring, $J$ be an ideal of $R$ and $M$ be a finitely generated $R$-module of dimension $t$. We denote by $\hat{R}$ the $\mathfrak{m}$-adic completion of $R$.

(1) Since $H_{J}^{t}(M)$ is an Artinian $R$-module, it also has the $\hat{R}$-module structure. In the proofs of [15, Theorem 2.4, Theorem 2.15], R. Sazeedeh showed that if the top local cohomology module $H_{J}^{t}(M)$ is strongly cotorsion as an $\hat{R}$-module, then it is strongly cotorsion as an $R$-module. Thus, from the point of view of studying Gorenstein injectivity of the top local cohomology module over a Gorenstein local ring, we shall assume that $R$ is always complete in the rest of this paper.

(2) If $R$ is complete and $H_{J}^{t}(M)$ is a nonzero strongly cotorsion $R$-module, then

$$
\operatorname{dim} R=\operatorname{dim} \Gamma_{\mathfrak{m}, J}\left(K_{M}\right) \leq \operatorname{dim} K_{M}=\operatorname{dim} M
$$

by Proposition 2.3 and [17, Lemma $1.9 \mathrm{c}$ ) ]. Thus, if $\operatorname{dim} M<\operatorname{dim} R$, then the nonzero top local cohomology module $H_{J}^{\operatorname{dim}} M(M)$ is not a strongly cotorsion $R$-module.

If $R$ is a Gorenstein local ring, then strongly cotorsion $R$-modules are precisely Gorenstein injective $R$-modules. Therefore it is natural to ask whether $H_{\mathfrak{m}}^{d}(R)$ is a Gorenstein injective $R$-module. This is however not true even if $R$ is a CohenMacaulay ring. In particular, the following assertion holds.

Theorem 2.6. Let $(R, \mathfrak{m})$ be a complete Cohen-Macaulay local ring of dimension $d$. Then the following conditions are equivalent:

(1) $R$ is Gorenstein;

(2) $H_{\mathfrak{m}}^{d}(R)$ is an injective $R$-module;

(3) $H_{\mathfrak{m}}^{d}(R)$ is a Gorenstein injective $R$-module.

Proof. The implications (1) $\Rightarrow(2)$ and $(2) \Rightarrow(3)$ are clear. Indeed, if $R$ is a Gorenstein local ring, then $H_{\mathfrak{m}}^{d}(R) \cong E_{R}(R / \mathfrak{m})$. We have only to prove $(3) \Rightarrow(1)$.

We assume that $H_{\mathfrak{m}}^{d}(R)$ is a Gorenstein injective $R$-module. Since $H_{\mathfrak{m}}^{d}(R)$ is Artinian, $H_{\mathfrak{m}}^{d}(R)^{\vee}$ is a finitely generated Gorenstein projective $R$-module by [ 5 , Theorem 4.8]. We note that the injective dimension of $H_{\mathfrak{m}}^{d}(R)^{\vee}$ is $d$. It follows from [11, Theorem 2.2] that $\operatorname{pd} H_{\mathfrak{m}}^{d}(R)^{\vee}=\operatorname{Gpd} H_{\mathfrak{m}}^{d}(R)^{\vee}=0$ where pd (resp. Gpd) is projective dimension (resp. Gorenstein projective dimension). Thus $H_{\mathfrak{m}}^{d}(R)^{\vee} \cong R^{n}$ for some integer $n$, so it follows that $H_{\mathfrak{m}}^{d}(R) \cong E(R / \mathfrak{m})^{n}$.

On the other hand, we have

$$
\operatorname{Hom}_{R}\left(H_{\mathfrak{m}}^{d}(R), H_{\mathfrak{m}}^{d}(R)\right) \cong \operatorname{Hom}_{R}\left(K_{R}, K_{R}\right) \cong R .
$$

(Note that since $R$ is a Cohen-Macaulay ring, it satisfies Serre's $\left(S_{2}\right)$-condition. This is used in the second isomorphism above. Also see [17, Theorem 1.14].) Thus the endomorphism ring $\operatorname{Hom}_{R}\left(H_{\mathfrak{m}}^{d}(R), H_{\mathfrak{m}}^{d}(R)\right)$ is a Noetherian local ring and finitely generated as an $R$-module. It follows from [18, Corollary 6.3] that $H_{\mathfrak{m}}^{d}(R)$ is an indecomposable $R$-module. Therefore we have $n=1$, so $K_{R} \cong R$. Consequently, we see that $R$ is Gorenstein.

The proof is completed. 
By Corollary 2.4 and Theorem 2.6, we get the following fact: In a complete Cohen-Macaulay local ring, the top local cohomology module $H_{\mathfrak{m}}^{d}(R)$ is nothing but one of the strongly cotorsion modules. But, if this module is a Gorenstein injective module, then all strongly cotorsion modules are Gorenstein injective.

Theorem 2.7. Let $(R, \mathfrak{m})$ be a complete Cohen-Macaulay local ring. Then the following conditions are equivalent:

(1) $R$ is Gorenstein;

(2) Strongly cotorsion modules are precisely Gorenstein injective modules.

Meanwhile, the following assertion holds.

Proposition 2.8. Let $(R, \mathfrak{m})$ be a complete Cohen-Macaulay local ring of dimension $d$. Then the following conditions are equivalent:

(1) $R$ is Artinian;

(2) $H_{\mathfrak{m}}^{d}(R)$ is a strongly torsion free $R$-module;

(3) $H_{\mathfrak{m}}^{d}(R)$ is a Gorenstein projective $R$-module;

(4) $H_{\mathfrak{m}}^{d}(R)$ is a Gorenstein flat $R$-module.

Proof. If $R$ is Artinian, then $d=0$ and $\Gamma_{\mathfrak{m}}(R)=R$. Thus implications (1) $\Rightarrow$ $(2),(3)$ and (4) are clear by each definition. Furthermore, in this situation, any Gorenstein projective $R$-module is Gorenstein flat (see [8, Corollary 3.4]). Hence we see that the implication $(3) \Rightarrow(4)$ holds. Therefore we have only to prove implications $(2) \Rightarrow(1)$ and $(4) \Rightarrow(1)$.

Let us prove $(2) \Rightarrow(1)$. Assume that $H_{\mathfrak{m}}^{d}(R)$ is a strongly torsion free $R$-module. Then $H_{\mathfrak{m}}^{d}(R)^{\vee} \cong K_{R}$ is a finitely generated strongly cotorsion $R$-module by [14, Lemma 2.1]. By [14, Theorem 3.4], $K_{R}$ is Artinian. This follows $\operatorname{dim} R=\operatorname{dim} K_{R}=$ 0 , so $R$ is Artinian.

Next, we shall show $(4) \Rightarrow(1)$. Assume that $H_{\mathfrak{m}}^{d}(R)$ is Gorenstein flat. Since fd $H_{\mathfrak{m}}^{d}(R)=d$, it follows from [10, Theorem 3.19] that we have $0=\operatorname{Gfd}_{\mathfrak{m}}^{d}(R)=$ $\mathrm{fd} H_{\mathfrak{m}}^{d}(R)=d$ where Gfd is Gorenstein flat dimension. Thus $R$ is Artinian.

The proof is completed.

Last of this section, we make a close examination of R. Sazeedeh's Theorem. We can see that the result of [15, Theorem 2.15] holds without assuming that a module is maximal Cohen-Macaulay.

Theorem 2.9. Let $(R, \mathfrak{m})$ be a complete Cohen-Macaulay local ring with dimension $d \leq 2$ and $M$ be a finitely generated $R$-module. Then $H_{J}^{d}(M)$ is a strongly cotorsion $R$-module for any ideal $J$ of $R$.

Proof. Suppose $d=2$. If $\operatorname{dim} M \leq 1$, then $H_{J}^{2}(M)=0$ by Grothendieck's vanishing theorem. In this case, $H_{J}^{2}(M)$ is strongly cotorsion. If $\operatorname{dim} M=2$, then $\operatorname{dim} K_{M}=$ $\operatorname{dim} M=2$. Since $R$ is a factor ring of a Gorenstein ring, $K_{M}$ satisfies Serre's $\left(S_{2}\right)$-condition (see [17, Lemma 1.9e]). Hence $K_{M}$ is a maximal Cohen-Macaulay $R$-module.

We consider a long exact sequence

$$
\begin{aligned}
0 \longrightarrow \Gamma_{\mathfrak{m}}\left(\Gamma_{\mathfrak{m}, J}\left(K_{M}\right)\right) \longrightarrow \Gamma_{\mathfrak{m}}\left(K_{M}\right) \longrightarrow \Gamma_{\mathfrak{m}}\left(K_{M} / \Gamma_{\mathfrak{m}, J}\left(K_{M}\right)\right) \\
\longrightarrow H_{\mathfrak{m}}^{1}\left(\Gamma_{\mathfrak{m}, J}\left(K_{M}\right)\right) \longrightarrow H_{\mathfrak{m}}^{1}\left(K_{M}\right) \longrightarrow H_{\mathfrak{m}}^{1}\left(K_{M} / \Gamma_{\mathfrak{m}, J}\left(K_{M}\right)\right) \\
\longrightarrow H_{\mathfrak{m}}^{2}\left(\Gamma_{\mathfrak{m}, J}\left(K_{M}\right)\right) \longrightarrow H_{\mathfrak{m}}^{2}\left(K_{M}\right) \longrightarrow H_{\mathfrak{m}}^{2}\left(K_{M} / \Gamma_{\mathfrak{m}, J}\left(K_{M}\right)\right) \longrightarrow 0 .
\end{aligned}
$$


Since $K_{M}$ is a maximal Cohen-Macaulay $R$-module, we have $\Gamma_{\mathfrak{m}}\left(K_{M}\right)=H_{\mathfrak{m}}^{1}\left(K_{M}\right)$ $=0$. Moreover, $\Gamma_{\mathfrak{m}}\left(K_{M} / \Gamma_{\mathfrak{m}, J}\left(K_{M}\right)\right) \subseteq \Gamma_{\mathfrak{m}, J}\left(K_{M} / \Gamma_{\mathfrak{m}, J}\left(K_{M}\right)\right)=0$ holds. Therefore we see that $H_{\mathfrak{m}}^{i}\left(\Gamma_{\mathfrak{m}, J}\left(K_{M}\right)\right)=0$ if $i \neq 2$. This means that $\Gamma_{\mathfrak{m}, J}\left(K_{M}\right)$ is a maximal Cohen-Macaulay $R$-module. It follows from Proposition 2.3 that $H_{J}^{2}(M)$ is a strongly cotorsion $R$-module.

We can show the case $\operatorname{dim} R=0,1$ by the same argument as above.

If the ring $R$ is Gorenstein, then the result of [13, Theorem 2.6] holds for any $R$-module. Thus the following result is also a generalization of [13, Theorem 2.7].

Corollary 2.10. Let $(R, \mathfrak{m})$ be a complete Gorenstein local ring with dimension $d \leq 2$ and $M$ be an $R$-module. Then $H_{J}^{d}(M)$ is a Gorenstein injective R-module for any ideal $J$ of $R$.

Proof. We may assume that $\operatorname{dim} M=d$. Let $M$ be an inductive limit $\lim _{\lambda \in \Lambda} N_{\lambda}$ of finitely generated $R$-submodules $N_{\lambda}$ of $M$. By Theorem 2.9, $H_{J}^{d}\left(N_{\lambda}\right)$ is a Gorenstein injective $R$-module. Since an inductive limit of Gorenstein injective modules over a Gorenstein local ring is also a Gorenstein injective module by [21, Theorem 5], $H_{J}^{d}(M)=H_{J}^{d}\left(\lim _{\lambda \in \Lambda} N_{\lambda}\right)=\lim _{\lambda \in \Lambda} H_{J}^{d}\left(N_{\lambda}\right)$ is Gorenstein injective.

\section{The Gorenstein InJECTIVITY of TOP LOCAL COHOMOLOGY MOdULES OVER GORENSTEIN RINGS}

In this section, we assume that all rings are Gorenstein. The main aim of this section is to give an example of a non-Gorenstein top local cohomology module over a 3-dimensional Gorenstein ring. To do this, we shall give a practical way of concluding whether top local cohomology modules are Gorenstein injective.

We begin with the following lemma.

Lemma 3.1. Let $I$ and $J$ be ideals of $R$ such that $0 \neq \Gamma_{I, J}(R) \subsetneq R$ and let $(0)=\bigcap_{i=1}^{m} \mathfrak{q}_{i}$ be an irredundant primary decomposition of the zero ideal of $R$. Then one has

$$
\Gamma_{I, J}(R)=\bigcap_{\sqrt{\mathfrak{q}_{i} \notin W(I, J)}} \mathfrak{q}_{i}
$$

Proof. Let $\sqrt{\mathfrak{q}_{i}}=\mathfrak{p}_{i}$ for each $i$. We may assume that $\mathfrak{p}_{1}, \ldots, \mathfrak{p}_{n} \in W(I, J)$ and $\mathfrak{p}_{n+1}, \ldots, \mathfrak{p}_{m} \notin W(I, J)$. By the definition of $W(I, J)$ there exists an integer $s>0$ such that $I^{s} \subseteq \prod_{i=1}^{n} \mathfrak{q}_{i}+J \subseteq \bigcap_{i=1}^{n} \mathfrak{q}_{i}+J$. It follows from $\bigcap_{i=1}^{n} \mathfrak{q}_{i} \subseteq \operatorname{Ann}_{R}\left(\bigcap_{i=n+1}^{m} \mathfrak{q}_{i}\right)$ that we have $I^{s} \subseteq \operatorname{Ann}_{R}\left(\bigcap_{i=n+1}^{m} \mathfrak{q}_{i}\right)+J$, and so we see that $\bigcap_{i=n+1}^{m} \mathfrak{q}_{i} \subseteq \Gamma_{I, J}(R)$.

Now, we consider a short exact sequence

$$
0 \rightarrow \bigcap_{i=n+1}^{m} \mathfrak{q}_{i} \rightarrow R \rightarrow R / \bigcap_{i=n+1}^{m} \mathfrak{q}_{i} \rightarrow 0 .
$$

Applying the left exact functor $\Gamma_{I, J}$ to this sequence, we get an exact sequence

$$
0 \rightarrow \Gamma_{I, J}\left(\bigcap_{i=n+1}^{m} \mathfrak{q}_{i}\right) \rightarrow \Gamma_{I, J}(R) \rightarrow \Gamma_{I, J}\left(R / \bigcap_{i=n+1}^{m} \mathfrak{q}_{i}\right) .
$$

Since $\bigcap_{i=1}^{m} \mathfrak{q}_{i}$ is irredundant, we see that

$$
\operatorname{Ass}\left(R / \bigcap_{i=n+1}^{m} \mathfrak{q}_{i}\right) \cap W(I, J)=\left\{\mathfrak{p}_{n+1}, \ldots, \mathfrak{p}_{m}\right\} \cap W(I, J)=\emptyset .
$$


Hence it follows that $\Gamma_{I, J}\left(R / \bigcap_{i=n+1}^{m} \mathfrak{q}_{i}\right)=0$ by [19, Proposition 1.10], so we have $\Gamma_{I, J}\left(\bigcap_{i=n+1}^{m} \mathfrak{q}_{i}\right)=\Gamma_{I, J}(R)$. Consequently, it follows that $\Gamma_{I, J}(R)=\bigcap_{i=n+1}^{m} \mathfrak{q}_{i}$.

For a given pair of ideals $I$ and $J$ of $R$, we consider the following ring.

Definition 3.2. Let $I$ and $J$ be ideals of $R$ such that $0 \neq \Gamma_{I, J}(R) \subsetneq R$ and let $(0)=\bigcap \mathfrak{q}_{i}$ be an irredundant primary decomposition of the zero ideal of $R$ with $\sqrt{\mathfrak{q}_{i}}=\mathfrak{p}_{i}$. Then we set

$$
R(I, J)=R / \bigcap_{\mathfrak{p}_{i} \notin W(I, J)} \mathfrak{q}_{i} .
$$

In the case $\Gamma_{I, J}(R)=0\left(\operatorname{resp} . \Gamma_{I, J}(R)=R\right)$, we set $R(I, J)=R(\operatorname{resp} . R(I, J)=$ $0)$.

If $R$ is a Cohen-Macaulay local ring of dimension $d$, then $R(I, J)$ is a zero or local ring of dimension $d$. But it is not necessarily a Cohen-Macaulay ring.

Recall that a local ring $R$ is called an almost Cohen-Macaulay ring if the CohenMacaulay defect $\mathrm{cmd} R=\operatorname{dim} R-\operatorname{depth} R$ is at most one. Now we are able to give a practical way of concluding that top local cohomology modules are Gorenstein injective.

Proposition 3.3. Let $(R, \mathfrak{m})$ be a complete Gorenstein local ring of dimension d and $J$ be an ideal of $R$. Then the following conditions are equivalent:

(1) $H_{J}^{d}(R)$ is a Gorenstein injective R-module;

(2) $R(\mathfrak{m}, J)$ is an almost Cohen-Macaulay ring.

Proof. We consider a short exact sequence

$$
0 \rightarrow \Gamma_{\mathfrak{m}, J}(R) \rightarrow R \rightarrow R(\mathfrak{m}, J) \rightarrow 0,
$$

and this implies a long exact sequence

$$
\begin{gathered}
0 \longrightarrow \Gamma_{\mathfrak{m}}\left(\Gamma_{\mathfrak{m}, J}(R)\right) \longrightarrow \Gamma_{\mathfrak{m}}(R) \longrightarrow \Gamma_{\mathfrak{m}}(R(\mathfrak{m}, J)) \\
\longrightarrow H_{\mathfrak{m}}^{1}\left(\Gamma_{\mathfrak{m}, J}(R)\right) \longrightarrow \quad \ldots .
\end{gathered}
$$

So we see that $\Gamma_{\mathfrak{m}}\left(\Gamma_{\mathfrak{m}, J}(R)\right)=\Gamma_{\mathfrak{m}}(R)$ and $H_{\mathfrak{m}}^{i}\left(\Gamma_{\mathfrak{m}, J}(R)\right) \cong H_{\mathfrak{m}}^{i-1}(R(\mathfrak{m}, J))$ for integers $i=1, \ldots, d-1$. It follows that $\Gamma_{\mathfrak{m}, J}(R)$ is a maximal Cohen-Macaulay module if and only if $R(\mathfrak{m}, J)$ is an almost Cohen-Macaulay ring. Since $R$ is a Gorenstein ring, the proof is completed by Proposition 2.3 ,

On the other hand, for a complete Gorenstein local ring $(R, \mathfrak{m})$ of dimension $d$ and an ideal $J$ of $R$, we can also characterize the Gorenstein injectivity of $H_{\mathfrak{m}, J}^{d}(R)$ by a ring $R(J, 0)$. There exists an isomorphism $H_{\mathfrak{m}, J}^{d}(R)^{\vee} \cong \Gamma_{J}(R)$ by [19, Corollary 5.12]. Since $\Gamma_{J}(R)$ is a finitely generated $R$-module and $H_{\mathfrak{m}, J}^{d}(R)$ is a submodule of $H_{\mathfrak{m}, J}^{d}(R)^{\vee \vee}, H_{\mathfrak{m}, J}^{d}(R)$ is an Artinian $R$-module. Thus, by the same argument as above, it is easy to see that the following generalization of [15, Theorem 2.4] holds.

Proposition 3.4. Let $(R, \mathfrak{m})$ be a complete Gorenstein local ring of dimension d and let $J$ be an ideal of $R$. Then the following conditions are equivalent:

(1) $H_{\mathfrak{m}, J}^{d}(R)$ is a Gorenstein injective R-module;

(2) $R(J, 0)$ is an almost Cohen-Macaulay ring. 
If the ring $R$ has the form $S /(f)$, where $S$ is a regular local ring and $f$ is an element of $S$, then $R$ is called a local hypersurface ring defined by $f$ in $S$.

The following result is an application of Proposition 3.3 .

Theorem 3.5. Let $(R, \mathfrak{m})$ be a complete local hypersurface ring with dimension d. Then $H_{J}^{d}(R)$ is a Gorenstein injective $R$-module for any ideal $J$ of $R$.

Proof. Let $R=S /(f)$, where $S$ is a regular local ring and $f \in S$. We shall show that $R(\mathfrak{m}, J)$ is also a complete local hypersurface ring.

Since a regular local ring is a UFD (a factorial domain in other terminology), $f$ can be expressed as a product of prime elements, that is,

$$
f=p_{1}^{n_{1}} p_{2}^{n_{2}} \cdots p_{s}^{n_{s}}
$$

where $p_{i}$ is a prime element in $S$ and $n_{i}$ is a positive integer for each $i=1, \ldots, s$. (It is a well-known fact that the factorization into prime elements is unique up to a unit element. We may also assume a condition $(*)$ which is $\left(p_{i}\right) \neq\left(p_{j}\right)$ if $i \neq j$.) Then

$$
(f)=\left(p_{1}^{n_{1}} p_{2}^{n_{2}} \cdots p_{s}^{n_{s}}\right)=\left(p_{1}^{n_{1}}\right) \cap\left(p_{2}^{n_{2}}\right) \cap \cdots \cap\left(p_{s}^{n_{s}}\right) .
$$

In fact, if $g \in \bigcap_{i=1}^{s}\left(p_{i}^{n_{i}}\right)$, then $p_{i}^{n_{i}}$ must appear in a unique factorization of $g$ for each $i$. By assumption (*) and since the prime element of $S$ is irreducible, this means that $g$ has $p_{1}^{n_{1}} p_{2}^{n_{2}} \cdots p_{s}^{n_{s}}$ in a unique factorization of itself, so $g \in\left(p_{1}^{n_{1}} p_{2}^{n_{2}} \cdots p_{s}^{n_{s}}\right)$.

Now we assume that $\left(p_{1}\right) R, \ldots,\left(p_{l}\right) R \in W(\mathfrak{m}, J)$ and $\left(p_{l+1}\right) R, \ldots,\left(p_{s}\right) R \notin$ $W(\mathfrak{m}, J)$. We note that $\left(\bigcap_{i=l+1}^{s}\left(p_{i}^{n_{i}}\right) R\right) \cap S=\bigcap_{i=l+1}^{s}\left(p_{i}^{n_{i}}\right)$. Indeed,

$$
\begin{aligned}
\bigcap_{i=l+1}^{s}\left(p_{i}^{n_{i}}\right) & =\left(\left(\bigcap_{i=l+1}^{s}\left(p_{i}^{n_{i}}\right)\right) R\right) \cap S \\
& \subseteq\left(\bigcap_{i=l+1}^{s}\left(p_{i}^{n_{i}}\right) R\right) \cap S \\
& =\bigcap_{i=l+1}^{s}\left(\left(p_{i}^{n_{i}}\right) R \cap S\right) \\
& =\bigcap_{i=l+1}^{s}\left(p_{i}^{n_{i}}\right) .
\end{aligned}
$$

Since $\operatorname{Ass}(R)=\left\{\left(p_{i}\right) R\right\}_{i=1, \cdots, s}$, we have

$$
R(\mathfrak{m}, J)=R / \bigcap_{i=l+1}^{s}\left(p_{i}^{n_{i}}\right) R=S / \bigcap_{i=l+1}^{s}\left(p_{i}^{n_{i}}\right) .
$$

By the same argument as above, we see that $\bigcap_{i=l+1}^{s}\left(p_{i}^{n_{i}}\right)=\left(p_{l+1}^{n_{l+1}} \cdots p_{s}^{n_{s}}\right)$. Thus we have

$$
R(\mathfrak{m}, J)=S /\left(p_{l+1}^{n_{l+1}} \cdots p_{s}^{n_{s}}\right)
$$

so $R(\mathfrak{m}, J)$ is a local hypersurface ring. In particular, $R(\mathfrak{m}, J)$ is a Cohen-Macaulay ring. Thus $H_{J}^{d}(R)$ is Gorenstein injective by Proposition 3.3.

Let $W$ be a subset of $\operatorname{Spec}(R)$. Recall that $W$ is said to be a specialization closed subset if $\mathfrak{p} \in W$ and $\mathfrak{p} \subseteq \mathfrak{q} \in \operatorname{Spec}(R)$ imply $\mathfrak{q} \in W$. We denote by $H_{W}^{i}$ the $i$ th right derived functor $\mathcal{R}^{i} \Gamma_{W}$ of the section functor $\Gamma_{W}$ with support in a specialization closed subset $W$ of $\operatorname{Spec}(R)$. 
Corollary 3.6. Let $R$ be a complete local hypersurface ring with dimension $d$. Then $H_{W}^{d}(R)$ is a Gorenstein injective $R$-module for any specialization closed subset $W$ of $\operatorname{Spec}(R)$.

Proof. In a Gorenstein local ring, an inductive limit of Gorenstein injective modules is also a Gorenstein injective module. (See [21, Theorem 5].) We note that $H_{W}^{d}(R)$ is an inductive limit of top local cohomology modules $H_{\mathfrak{a}}^{d}(R)$. In fact, in a similar manner to [19, Theorem 3.2], we can prove that there is a natural isomorphism

$$
H_{W}^{i}(R) \cong \underset{\mathfrak{a} \in \widetilde{W}}{\lim _{\mathfrak{W}}} H_{\mathfrak{a}}^{i}(R)
$$

for any integer $i$, where $\tilde{W}$ is the set of ideals $\mathfrak{a}$ of $R$ such that $\Gamma_{W}(R / \mathfrak{a})=R / \mathfrak{a}$. (Also see M. H. Bijan-Zadeh's paper [1, Proposition 2.3].) Thus $H_{W}^{d}(R)$ is Gorenstein injective by Theorem 3.5 .

Finally, we give two examples of a top local cohomology module: one is Gorenstein injective but not injective, and the other is non-Gorenstein injective.

Example 3.7. Let $k$ be a field.

(1) Let $R=k[[x, y, z, w]] /\left(x y^{2}\right), \mathfrak{m}$ be a maximal ideal of $R$ and $J=(y, z, w) R$. $R$ is a hypersurface and 3-dimensional complete Gorenstein local ring. We have a primary decomposition $\left(x y^{2}\right)=(x) \cap\left(y^{2}\right)$ and $\operatorname{Ass}(R)=\{(x) R,(y) R\}$. We note that $(x) R+J$ is an $\mathfrak{m}$-primary ideal and $(y) R+J$ is not an $\mathfrak{m}$-primary ideal. Thus $\operatorname{Ass}(R) \cap W(\mathfrak{m}, J)=\{(x) R\}$, and so $R(\mathfrak{m}, J)=k[[x, y, z, w]] /\left(y^{2}\right)$. This is a Cohen-Macaulay ring. Consequently, $H_{J}^{3}(R)$ is a Gorenstein injective $R$-module.

But this module is not injective. In fact, we suppose that $H_{J}^{3}(R)$ is an injective $R$-module. Since $H_{J}^{3}(R)$ is Artinian, $H_{J}^{3}(R)^{\vee}$ is a finitely generated flat $R$-module. In particular, $H_{J}^{3}(R)^{\vee}$ is a finitely generated free $R$-module $R^{n}$ for some integer $n$. Let an $R$-isomorphism be

$$
\varphi: R^{n} \stackrel{\sim}{\longrightarrow} H_{J}^{3}(R)^{\vee} \stackrel{\sim}{\longrightarrow} \Gamma_{\mathfrak{m}, J}(R)=\left(y^{2}\right) R .
$$

Then it follows that $0 \neq \varphi\left(x \cdot R^{n}\right)=x \cdot \varphi\left(R^{n}\right)=x \cdot\left(y^{2}\right) R=0$. This is a contradiction.

Therefore the top local cohomology module

$$
H_{(y, z, w)}^{3}\left(k[[x, y, z, w]] /\left(x y^{2}\right)\right)
$$

is a Gorenstein injective but not an injective $R$-module.

(2) Let $R=k[[x, y, z, u, v, w]] /(x u, y v, z w), \mathfrak{m}$ be a maximal ideal of $R$ and $J=(x+y, y+z, u+v, v+w) R . R$ is a 3 -dimensional complete intersection local ring but not a hypersurface.

A primary decomposition of $(x u, y v, z w)$ is

$$
\begin{aligned}
& (x, y, z) \cap(x, y, w) \cap(x, z, v) \cap(x, v, w) \\
& \quad \cap(y, z, u) \cap(y, u, w) \cap(z, u, v) \cap(u, v, w),
\end{aligned}
$$

and

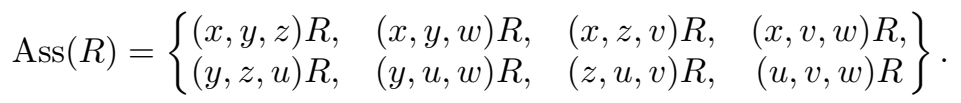

$(x, y, z) R$ and $(u, v, w) R$ are two associated prime ideals $\mathfrak{p}$ of $R$ such that $\mathfrak{p}+J$ is not an $\mathfrak{m}$-primary ideal. Thus $\operatorname{Ass}(R) \cap W(\mathfrak{m}, J)=\operatorname{Ass}(R) \backslash\{(x, y, z) R,(u, v, w) R\}$, and so

$$
R(\mathfrak{m}, J)=k[[x, y, z, u, v, w]] /(x, y, z) \cap(u, v, w) .
$$


Then $\operatorname{dim} R(\mathfrak{m}, J)=3$ and $\operatorname{depth} R(\mathfrak{m}, J)=1$, so $R(\mathfrak{m}, J)$ is not an almost CohenMacaulay ring. Therefore it follows from Proposition 3.3 that the top local cohomology module

$$
H_{(x+y, y+z, u+v, v+w)}^{3}(k[[x, y, z, u, v, w]] /(x u, y v, z w))
$$

is not a Gorenstein injective $R$-module.

In fact, we can also see that there exists an $R$-module $X$ with finite flat dimension such that $\operatorname{Ext}_{R}^{1}\left(X, H_{J}^{3}(R)\right) \neq 0$ as follows. (Also see Remark 1.3 (2).) We take a maximal regular sequence $x+u, y+v, z+w$ on $R$ and set $\mathfrak{n}=(x+u, y+v, z+$ $w) R$. Since $\mathfrak{n}$ is an $\mathfrak{m}$-primary ideal and $\operatorname{depth} R(\mathfrak{m}, J)=1$, it is easy to see that $\operatorname{grade}\left(\mathfrak{n}, \Gamma_{\mathfrak{m}, J}(R)\right)=2$. So it follows that $\operatorname{Ext}_{R}^{2}\left(R / \mathfrak{n}, \Gamma_{\mathfrak{m}, J}(R)\right) \neq 0$. We also note that $\operatorname{fd}_{R}(R / \mathfrak{n})=3$. Then we have

$$
\begin{aligned}
\operatorname{Ext}_{R}^{1}\left(R / \mathfrak{n}, H_{J}^{3}(R)\right) & \cong \operatorname{Ext}_{R}^{1}\left(R / \mathfrak{n}, \Gamma_{\mathfrak{m}, J}(R)^{\vee}\right) \\
& \cong \operatorname{Tor}_{1}^{R}\left(R / \mathfrak{n}, \Gamma_{\mathfrak{m}, J}(R)\right)^{\vee} \\
& \cong \operatorname{Ext}_{R}^{2}\left(R / \mathfrak{n}, \Gamma_{\mathfrak{m}, J}(R)\right)^{\vee} \neq 0 .
\end{aligned}
$$

(Note that [12, Theorem 2] is used in the last isomorphism above.)

Thus R. Sazeedeh's Theorem for arbitrary dimension does not hold.

Remark 3.8. By the same argument as in Example 3.7 (1), we can also see that there exists a hypersurface ring $R$ with dimension $d \leq 2$ and an ideal $J$ of $R$ such that $H_{J}^{d}(R)$ is not an injective $R$-module. For example, for a 2-dimensional complete local hypersurface ring $R=k[[x, y, z]] /(y z)$ and $J=(x, y) R$, the top local cohomology module $H_{J}^{2}(R) \cong \Gamma_{(x, y, z) R, J}(R)^{\vee}=((y) R)^{\vee}$ is not an injective $R$-module.

\section{ACKNOWLEDGMENTS}

The author expresses gratitude to the referees for their kind comments and valuable suggestions. Thanks to the referees' advice, the author could add Remark 3.8 to the earlier version of this paper.

\section{REFERENCES}

1. M. H. Bijan-Zadeh, Torsion theories and local cohomology over commutative and Noetherian rings, J. Lond. Math. Soc. 19 (2), no. 3 (1979), 402-410. MR.540052 (80h:13013)

2. M. P. Brodmann and R. Y. Sharp, Local cohomology: An algebraic introduction with geometric applications, Cambridge University Press, Cambridge (1998). MR.1613627(99h:13020)

3. L. W. Christensen, Gorenstein Dimension, Lecture Notes in Mathematics 1747, SpringerVerlag, Berlin (2000). MR,1799866 (2002e:13032)

4. L. W. Christensen, H.-B. Foxby and A. Frankild, Restricted homological dimensions and Cohen-Macaulayness, J. Algebra 251, no. 1 (2002), 479-502. MR.1900297 (2003e:13022)

5. E. E. Enochs and O. M. G. Jenda, On Gorenstein injective modules, Comm. Algebra 21, no. 10 (1993), 3489-3501. MR1231612 (94g:13006)

6. E. E. Enochs and O. M. G. Jenda, Gorenstein injective and projective modules, Math. Z. 220, no. 4 (1995), 611-633. MR1363858 (97c:16011)

7. E. E. Enochs, O. M. G. Jenda and B. Torrecillas, Gorenstein flat modules, Nanjing Daxue Xuebao Shuxue Bannian Kan 10, no. 1 (1993), 1-9. MR1248299 (95a:16004)

8. E. E. Enochs, O. M. G. Jenda and J. Xu, Foxby duality and Gorenstein injective and projective modules, Trans. Amer. Math. Soc. 348, no. 8 (1996), 3223-3234. MR.1355071 (96k:13010)

9. A. A. Gerko, On homological dimension, Mat. Sb. 192, no. 8 (2001), 79-94. MR1862245 (2002h:13024) 
10. H. Holm, Gorenstein homological dimensions, J. Pure Appl. Algebra 189, no. 1-3 (2004), 167-193. MR2038564 (2004k:16013)

11. H. Holm, Rings with finite Gorenstein injective dimension, Proc. Amer. Math. Soc. 132, no. 5 (2004), 1279-1283. MR2053331 (2005a:13031)

12. E. Matlis, The higher properties of R-sequences, J. Algebra 50, no. 1 (1978), 77-112. MR 479882 (80a:13013)

13. R. Sazeedeh, Gorenstein injective modules and local cohomology, Proc. Amer. Math. Soc. 132, no. 10 (2004), 2885-2891. MR2063107 (2005e:13027)

14. R. Sazeedeh, Strongly torsion free, copure flat and Matlis reflexive modules, J. Pure Appl. Algebra 192, no. 1-3 (2004), 265-274. MR2067199 (2005c:13008)

15. R. Sazeedeh, Strongly torsion-free modules and local cohomology over Cohen-Macaulay rings, Comm. Algebra 33, no. 4 (2005), 1127-1135. MR2136689 (2006c:13015)

16. P. Schenzel, Explicit computations around the Lichtenbaum-Hartshorne vanishing theorem, Manuscripta Math. 78, no. 1 (1993), 57-68. MR1201761 (93m:14003)

17. P. Schenzel, On the use of local cohomology in algebra and geometry, in: Lectures at the Summer School of Commutative Algebra and Algebraic Geometry, Ballaterra, Birkhäuser, Basel (1996). MR.1648667 (99k:13025)

18. P. Schenzel, On connectedness and indecomposibility of local cohomology modules, Manuscripta Math. 128, no. 3 (2009), 315-327. MR2481047(2010a:13030)

19. R. Takahashi, Y. Yoshino and T. Yoshizawa, Local cohomology based on a nonclosed support defined by a pair of ideals, J. Pure Appl. Algebra 213, no. 4 (2009), 582-600. MR 2483839 (2009m:13027)

20. J. Xu, Minimal injective and flat resolutions of modules over Gorenstein rings, J. Algebra 175, no. 2 (1995), 451-477. MR1339651 (96h:13025)

21. O. Yi, On torsion Gorenstein injective modules, Arch. Math. (Brno) 34, no. 4 (1998), 445-454. MR:1679639 (2000h:16006)

Graduate School of Natural Science and Technology, Okayama University, Okayama 700-8530, JAPAN

E-mail address: tyoshiza@math.okayama-u.ac.jp 